\title{
A Very Rare Cause of Splenic Injury: Splenic Hematoma Following a Diagnostic Upper Endoscopy (Esophagogastroduodenoscopy) and Biopsy of Gastric Ulcer
}

\author{
Ahmad Eter $^{\mathrm{a}, \mathrm{c}}$, Rachel Belliveau ${ }^{\mathrm{b}}$
}

\begin{abstract}
The potential for splenic injury from esophagogastroduodenoscopy (EGD) is exceptionally low. To our knowledge, less than five cases have been reported in literature. Though still uncommon, splenic rupture due to diagnostic or therapeutic procedures is more frequently described following colonoscopy and endoscopic retrograde cholangiopancreatography. We report a 62-year-old Caucasian male with primary squamous cell carcinoma of the lung who presented 2 days after an EGD and gastric ulcer biopsy. The patient complained of severe left upper quadrant abdominal pain with rebound tenderness. Upon further evaluation, a diagnosis of a large subcapsular splenic hematoma was made with computed tomography (CT) imaging. The mechanism of splenic injury attributable to EGD is traction on the greater curvature of the stomach, causing avulsion of the splenic or short gastric vessels. We feel this case represents a very rare yet serious complication of EGD that warrants consideration and further investigation.
\end{abstract}

Keywords: EGD; Spleen; Hematoma; Metastatic gastric ulcer; Greater curvature; Injury

\section{Introduction}

Esophagogastroduodenoscopy (EGD) is a powerful and routinely implemented tool in evaluation of the upper gastrointestinal tract. Though many commonly acknowledged

Manuscript submitted March 12, 2020, accepted March 23, 2020

aDepartment of Internal Medicine, Princeton Community Hospital, 122 12th St, Princeton, WV 24740, USA

bWest Virginia School of Osteopathic Medicine, 400 N Lee St, Lewisburg, WV 24901, USA

${ }^{\mathrm{c} C o r r e s p o n d i n g ~ A u t h o r: ~ A h m a d ~ E t e r, ~ D e p a r t m e n t ~ o f ~ I n t e r n a l ~ M e d i c i n e, ~}$ Princeton Community Hospital, 122 12th St, Princeton, WV 24740, USA. Email: ahmadeter1@hotmail.com

doi: https://doi.org/10.14740/jocmr4134 adverse events such as difficulty swallowing and abdominal discomfort are typically mild, splenic injury is an exceedingly rare and serious complication. While iatrogenic endoscopic splenic injury has been infrequently described following endoscopic retrograde cholangiopancreatography (ERCP) and colonoscopy, only a few reported cases have been attributed to EGD [1-4]. Sharp abdominal pain, hemodynamic instability, and signs of occult hemorrhage can indicate splenic rupture. However, the patient may not present with signs and symptoms of splenic injury at the conclusion of the EGD, leading to potential delay in diagnosis [2]. It is imperative that splenic injury is considered after EGD as failure of early recognition and treatment can result in significant morbidity and mortality.

\section{Case Report}

A 62-year-old Caucasian male with primary squamous cell carcinoma of the lung and chronic anemia presented with generalized weakness and shortness of breath. The patient was cachectic with a body mass index (BMI) of 15.6, mildly tachycardic, tachypneic, and had an unremarkable abdominal exam. The patient was found to have a hemoglobin of $6.0 \mathrm{~g} / \mathrm{dL}$ (decreased from patient's baseline hemoglobin of $10.3 \mathrm{~g} / \mathrm{dL}$ ), hematocrit of $31.6 \%$ (decreased from patient's baseline of 35\%), and positive fecal occult blood test. Two units of packed red blood cells (PRBC) were transfused, bringing the hemoglobin to $7.3 \mathrm{~g} / \mathrm{dL}$ and the hematocrit to $21.1 \%$.

The patient underwent a technically simple EGD to evaluate the gastrointestinal bleed. The patient was placed in the routine left lateral position. A $2 \mathrm{~cm}$ ulcer with raised margins and trace blood was identified on the lower body of the stomach. Three biopsies were taken which confirmed metastasis from the lung. Immediately post procedure the patient's vitals were within normal limits. Repeat hemoglobin and hematocrit were $7.4 \mathrm{~g} / \mathrm{dL}$ and $21.6 \%$ respectively, prompting transfusion of one additional unit of PRBC. The patient had no signs of active gastrointestinal bleeding.

Forty-eight hours later the patient presented with severe left upper quadrant abdominal pain and rebound tenderness. The patient was visibly distressed and tachypneic at 22 respi- 

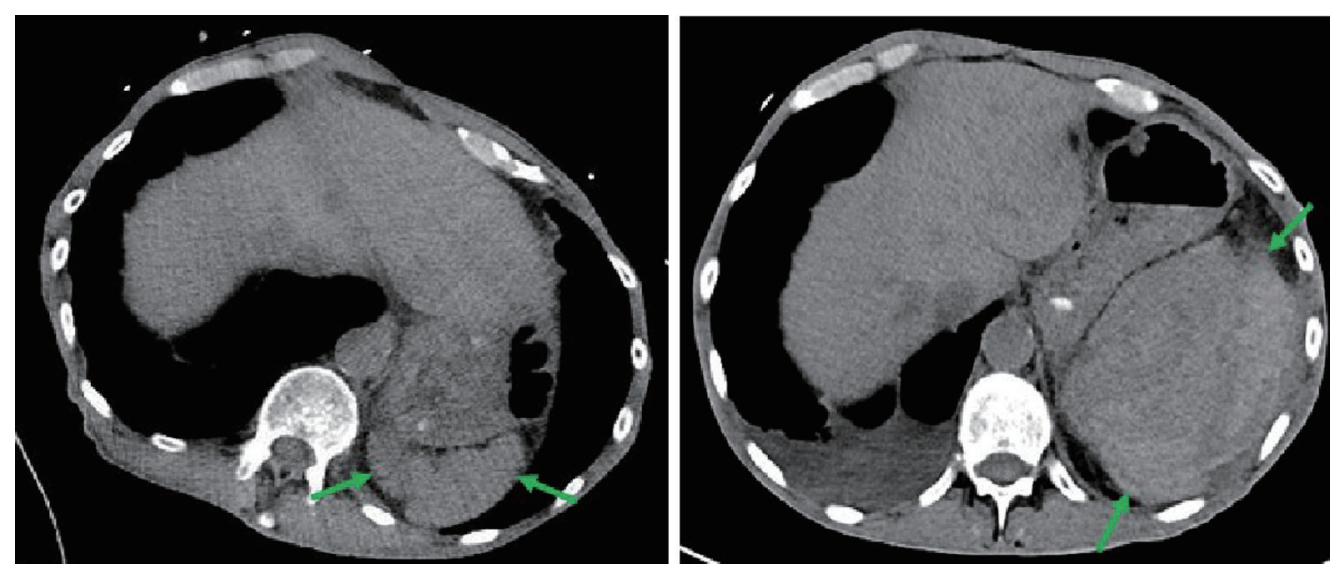

Figure 1. CT revealing a normal spleen 4 days prior to EGD (left) and an enlarged spleen with a subcapsular hematoma $48 \mathrm{~h}$ after the EGD (right). CT: computed tomography; EGD: esophagogastroduodenoscopy.

rations per minute. Notable laboratory values include hemoglobin of $9.3 \mathrm{~g} / \mathrm{dL}$ and hematocrit $26.7 \%$, decreased mildly from $9.8 \mathrm{~g} / \mathrm{dL}$ and $28.7 \%$ respectively.

An abdominal computed tomography (CT) was significant for an enlarged spleen measuring $14 \times 13 \times 9 \mathrm{~cm}$ compared to $8 \times 8.1 \times 3.3 \mathrm{~cm}$ on a recent scan. The findings were consistent with a large splenic hematoma with medial compression of normal splenic parenchyma (Fig. 1). No active bleeding, splenic infarcts, or signs of splenic metastasis were noted at the time of the imaging.

The patient did not have a history of anticoagulation therapy, trauma to the spleen, or diseases with known splenic involvement. As he was hemodynamically stable, conservative medical management was initiated with pantoprazole, morphine sulfate, oxygen, hemodynamic monitoring, sequential hemoglobin and hematocrit measurements, and serial abdominal exams. After conservative medical management, our patient showed marked clinical improvement in his symptoms and did not warrant further intervention.

\section{Discussion}

Sharp abdominal pain or signs of hemodynamic instability following EGD should raise suspicion for splenic injury, even if the symptoms begin days after the EGD. In addition to clinical and laboratory evaluation, a CT scan is the gold standard for suspected splenic injury [5]. The exact mechanism of splenic injury consequential to EGD is unknown. It has been suggested that bowing of the endoscope along the greater curvature of the stomach can cause avulsion of the splenic or short gastric vessels. The strain can be so great as to tear the splenic capsule, and may be magnified by insufflation of the stomach [2, $3,6,7]$. Direct splenic injury can also result from manipulation of the endoscope, especially when larger caliber scopes such as the Olympus GF-UM3 and TJFV10 are utilized [2]. Other factors that might predispose to splenic injury from EGD are coagulopathies, cirrhosis, abdominal adhesions, and pancreatitis [6]. In addition to endoscopic trauma, iatrogenic splenic hematomas and rupture can result from lithotripsy or left-sided thoracentesis. Non-iatrogenic causes of splenic insult include abdominal trauma, malignancy, infections (notably EpsteinBarr virus (EBV) and malaria), pregnancy, and metabolic and connective tissue disorders [1].

Our patient's initial anemia is attributable to acute gastrointestinal blood loss from a malignant gastric ulcer. However, the patient developed concerning clinical symptoms of left upper quadrant pain and a mild decrease in hemoglobin after the EGD, without signs of active gastrointestinal hemorrhage. Given the temporal relationship to the EGD and having eliminated other known causes from our differential, it is our belief that our patient's subcapsular splenic hematoma could be from the EGD. We believe our patient's greatest risk factor was the metastatic gastric ulcer on the greater curvature of the stomach. The short gastric and splenic vessels were likely compromised by the ulcer's proximity, thus vulnerable to the bowing of the endoscope.

Splenic injury may be managed conservatively with strict monitoring of heart rate, blood pressure, hemoglobin, and hematocrit. Conservative management is more likely to be successful if the splenic hilum is intact, even in the presence of capsule rupture [8]. Signs of hemodynamic instability warrant laparotomy or angiographic embolization followed by splenectomy; splenectomy is the definitive treatment for splenic rupture $[5,7]$.

\section{Conclusions}

Though exceptionally rare, splenic injury is a possible adverse event following EGD. Signs of acute abdominal pain or hemodynamic instability, even days after EGD, should prompt suspicion of splenic injury. We urge practitioners to consider this adverse event, as delayed diagnosis may have severe consequences.

\section{Acknowledgments}

None to declare. 


\section{Financial Disclosure}

None to declare.

\section{Conflict of Interest}

None to declare.

\section{Informed Consent}

Not applicable.

\section{Author Contributions}

Dr. AE identified the case, contributed to the conception and design, reviewed the literature, contributed to the manuscript draft, and made final approval of the version to be published. $\mathrm{RB}$ reviewed the literature, drafted the manuscript, and submitted for publishing. Both authors acquired and analyzed the data.

\section{Data Availability}

The authors declare that data supporting the findings of this study are available within the article.

\section{References}

1. Estevez-Boullosa P, Alonso-Aguirre PA, Couto-Worner I, Blanco-Rodriguez M, de Llano-Monelos P, Sanchez-Gonzalez F. Splenic rupture following a diagnostic upper endoscopy. World J Gastrointest Endosc. 2010;2(6):235-236.

2. Lewis FW, Moloo N, Stiegmann GV, Goff JS. Splenic injury complicating therapeutic upper gastrointestinal endoscopy and ERCP. Gastrointest Endosc. 1991;37(6):632-633.

3. Mazulis A, Lakha A, Qazi B, Shapiro A. Delayed presentation of splenic rupture after endoscopy in a patient with hemophilia a: case report and review of the literature. ACG Case Rep J. 2014;1(4):175-177.

4. Hunter RC, Jr. Gastroscopy and delayed rupture of the spleen; a review and report of a possible case. Gastroenterology. 1955;29(5):898-906.

5. Montenovo M, Javed E, Bakthavatsalam R, Reyes J. Splenic Subcapsular Hematoma After Endoscopic Retrograde Cholangiopancreatography in a Liver Transplant Recipient: Case Report and Literature Review. Exp Clin Transplant. 2017;15(1):103-105.

6. Chavalitdhamrong D, Donepudi S, Pu L, Draganov PV. Uncommon adverse events of ERCP. Digestive Endoscopy. 2014; 26:15-22.

7. Zyromski NJ, Camp CM. Splenic injury: a rare complication of endoscopic retrograde cholangiopancreatography. Am Surg. 2004;70(8):737-739.

8. Khan S, Yousuf MI, Bisharat M, Wali J. Iatrogenic splenic injury in percutaneous procedures. Ulster Med J. 2008;77(2):131-132. 